\title{
Progression of Jackhammer Esophagus to Achalasia
}

TO THE EDITOR: Abdallah and Fass ${ }^{1}$ recently reported an interesting case of a patient with dysphagia who was initially diagnosed with Jackhammer esophagus (JE) and progressed to type 2 achalasia over the course of approximately 2 years. We read this report with great interest as our group has studied the natural course of $\mathrm{JE}$ and has also found progression of JE to achalasia, which we reported previously in $2014 .^{2}$ In our case series, the single most important risk factor for progression of $\mathrm{JE}$ to achalasia was an elevated integrated relaxation pressure (IRP) on the initial manometry.

Though the reported patient's initial manometry showed a normal IRP a barium swallow at the time displayed a narrowed gas- troesophageal junction. One common pitfall in measuring the IRP in $\mathrm{JE}$ is an upward displacement of the lower esophageal sphincter (LES) due to esophageal shortening caused by vigorous contractions after swallows. Therefore, during swallows, the LES does not remain at the same level of resting pressure measurement and adjustments must be made in order to accurately calculate the IRP. An example of this phenomenon is shown in Figures 1 and 2. On further evaluation of Figure 2 by Abdallah and Fass, ${ }^{1}$ we believe that the IRP may have been indeed elevated on the initial manometry. It appears that the IRP was measured below the actual location of the LES at the time of swallow, thereby artificially lowering the
A

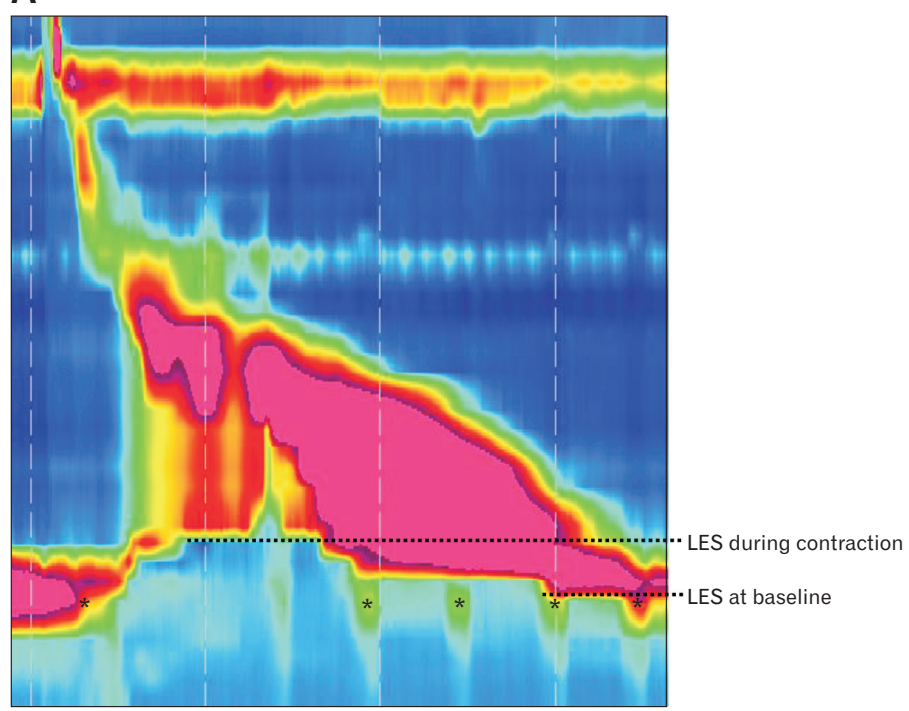

B

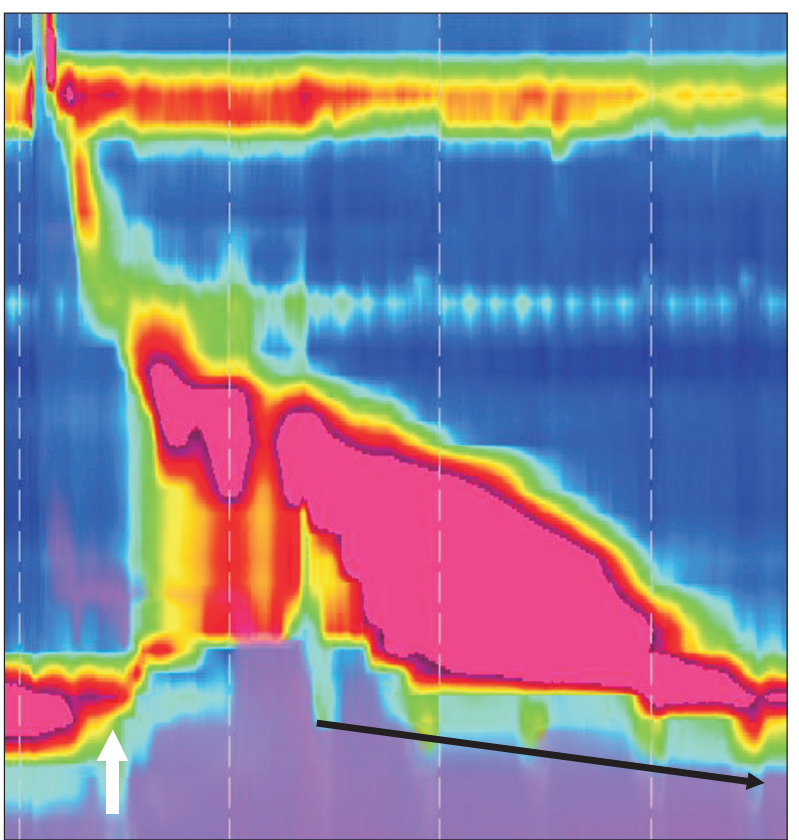

Figure 1. Esophageal shortening and upward displacement of lower esophageal sphincter (LES) during a Jackhammer contraction. (A) Jackhammer contraction with a distal contractile integral (DCI) of $38710 \mathrm{mmHg} \cdot \mathrm{cm} \cdot \mathrm{sec}$. During the contraction, the esophagus shortens by $3 \mathrm{~cm}$ which can be seen by separation of the LES (dotted line) from the diaphragmatic crura (ie, the pressure inversion point [*]) and subsequently returns to original resting position. (B) Further proof of esophageal shortening with impedance. Low impedance signal in the stomach corresponding to the level of saline rises during the esophageal contraction/shortening (white arrow) and slowly returns back to the original location as contraction dissipates (black arrow). 


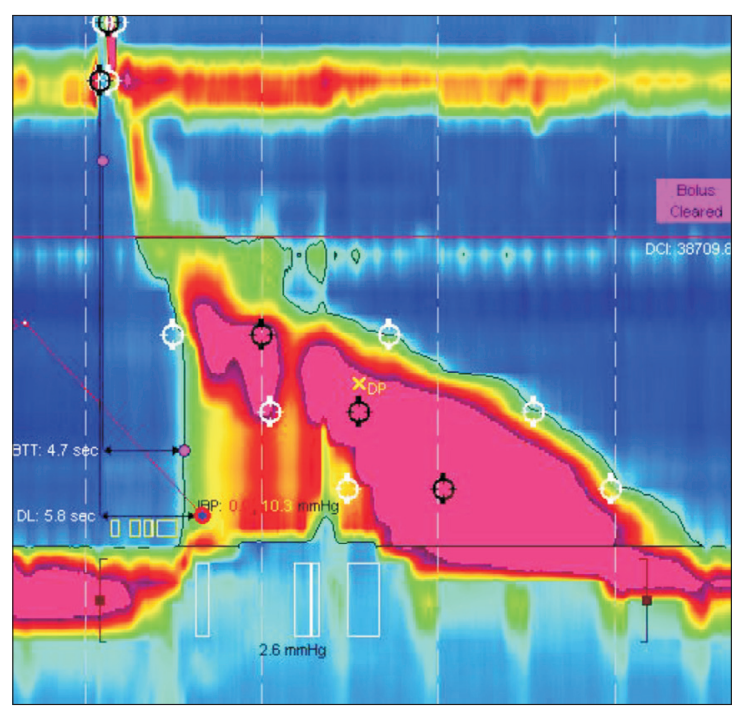

Figure 2. Measurement of the integrated relaxation pressure (IRP) using the original location of the lower esophageal sphincter (LES) will show a falsely normal level of $2.6 \mathrm{mmHg}$. After adjusting the LES by $3 \mathrm{~cm}$, the IRP was calculated as $48 \mathrm{mmHg}$ which is highly abnormal.

IRP measurement. This finding would correspond to the narrowed gastroesophageal junction seen on the initial barium swallow as well.

In our study, we found that patients with JE and elevated IRP who do not meet the criteria for achalasia at the time of JE diagnosis progressed to type 3 achalasia over approximately 2 years. As type 2 and type 3 achalasia may not share similar histopathologic origins, it would be interesting to study whether JE has a higher tendency to progress to either type of achalasia or even achalasia type $1 .{ }^{3,4}$ Future longitudinal studies are needed to elaborate the natural manometric progression of JE.

Lizhou Huang $^{1}$ and Ali Rezaie ${ }^{2}$

${ }^{\prime}$ Department of Medicine, Cedars-Sinai Medical Center, Los Angeles, CA, USA; and ${ }^{2}$ Division of Gastroenterology, Cedars-Sinai Medical Center, Los Angeles, CA, USA

1. Abdallah J, Fass R. Progression of Jackhammer esophagus to type II achalasia. J Neurogastroenterol Motil 2016;22:153-156.

2. Huang L, Rezaie A, Basseri B, Chang C, Pimentel M. Natural manometric course of Jackhammer esophagus and its determinants; a largescale database analysis. Gastroenterology 2014;146(suppl 1):S679.

3. Kahrilas PJ, Boeckxstaens G. The spectrum of achalasia: lessons from studies of pathophysiology and high-resolution manometry. Gastroenterology 2013;145:954-965.

4. Sodikoff JB, Lo AA, Shetuni BB, Kahrilas PJ, Yang GY, Pandolfino JE. Histopathologic patterns among achalasia subtypes. Neurogastroenterol Motil 2016;28:139-145.

\section{Conflicts of interest: None.}

